\title{
Neurotoxicity of Drugs in Children During Antenatal and Postnatal Development Periods
}

\author{
Sergey Postnikov ${ }^{1,2}$, Natalia Teplova ${ }^{1}$, Aleksey Ermilin ${ }^{1,2}$, Marya Kostyleva ${ }^{1,2}$, \\ Anna Gratzhianskaya ${ }^{1,2}$, Galina Chervyakova ${ }^{1}$, Yulia Eremina ${ }^{1}$ \\ ${ }^{1}$ Chair of Clinical Pharmacology, Russian National Research Medical University Named After Pirogov, Moscow, Russian Federation \\ ${ }^{2}$ Russian Children Clinical Hospital of Ministry of Health, Moscow, Russian Federation
}

\section{Email address:}

salmova@mail.ru (S. Postnikov), Teplova.nv@yandex.ru (N. Teplova), elixin@list.ru (A. Ermilin),

Kostyleva_m_n@rdkb.ru (M. Kostyleva), annagrats@rambler.ru(A. Gratzhianskaya)

\section{To cite this article:}

Sergey Postnikov, Natalia Teplova, Aleksey Ermilin, Marya Kostyleva, Anna Gratzhianskaya, Galina Chervyakova, Yulia Eremina. Neurotoxicity of Drugs in Children During Antenatal and Postnatal Development Periods. American Journal of Pediatrics.

Vol. 6, No. 1, 2020, pp. 62-67. doi: 10.11648/j.ajp.20200601.21

Received: November 8, 2019; Accepted: February 14, 2020; Published: February 28, 2020

\begin{abstract}
Neurotoxicity is not rare and periodically severe and even life-threatening adverse effect (AE) of drugs belonging to many therapeutic groups. Manifestations of neurotoxicity are variable: disturbances of peripheral nervous system (ototoxicity and ophtalmotoxicity, visceral neuropathy, neuromuscular blockade), involving of central nervous system (CNS) (developmental brain malformations, seizures, impairment of consciousness, nonspecific encephalopathy). In the way of its consequences central nervous system damage is the most severe manifestation of drug neurotoxicity but the main predisposing factor is related to immature but growing intensively child brain of first years of life; the primary factor for neurotoxic effect is the capacity of drugs to transfer through brain-blood barrier and cumulate into CNS. The principal mechanism of neurotoxicity is dysfunction/apoptosis of nervous cells. Aim of this survey is to provide data and attract once again the professionals attention to the neurological complications of those drugs in the first place that are mainly targeted to the CNS effects (anticonvulsants, antidepressants, antipsychotic drugs, anesthetics) among the mostly sensitive groups - pregnant women, newborns, infants. Results. Based on analyzed data it was revealed high incidence of CNS complications both transient (seizures, temporarily impairment of consciousness) and persistent (developmental brain malformations, decrease of cognitive functions) in case of use of the drugs of current interest (anticonvulsants, antidepressants, antipsychotics, anesthetics (general and local), antibiotics) in risk groups - fetus, newborns, infants. Population risk of neurotoxicity includes also ethnic and genetic special features, renal and hepatic failure, background neuropsychic diseases except determined age groups. Conclusion. Neurotoxicity is one of the most severe manifestations of drug intolerance that depends on different aspects. The youngest targeted group is children of $0-3$ years old due to their developmental immature brain and age-dependent specific characteristics of pharmacodynamics and pharmacokinetics that could result in drug overdose as a particular case of neurotoxicity due underestimation of the mentioned above factors.
\end{abstract}

Keywords: Neurotoxicity, Anticonvulsants, Psychotropic Agents, Anesthetics, Antibiotics, Pregnancy, Lactation

\section{Introduction}

The history of neurotoxicity of drugs has started from the middle of XIX century when Huette described the side effect of bromides as sedation, decreased cognition and behavioral disturbances.

Since that time the attention to this problem is only increased firstly due to its importance and secondly due to enormous growth of pharmaceutical market and numerous drugs for which neurotoxic effect was revealed - antibiotics, glucocorticoids, antiviral drugs, statins, antipsychotic drugs, anticonvulsants, anesthetics [1-5].

The total incidence of drugs neurotoxicity could not be subject to a determination due to enormous gap between description of separate cases like generalized seizures in case of lidocaine overdose [5] up to significant incidence of depressive disorders (40\%) and suicidal thoughts (47\%) in case of anticonvulsants [6]. The global statistics is also compromised by variety of manifestations of drugs neurotoxicity: modification of taste, injury 
of visual nerve, delirium, psychotic reactions and rather high level of mortality (12-26\%) because of neurological complications mostly caused by hemorrhage or ischemia of brain in patients after bone marrow transplantation $(64.7 \%)[7,8]$.

\section{Predisposing Factors}

In the first place it's capacity of drug to penetrate and accumulate in central nervous system (CNS) that depends on hematoencephalic barrier (HEB) evaluating in particular by expressing (genetic polymorphism) of transporter of organic anions (OAT 1,3 and 1A2), by lipophilic characteristics of the drug (ratio of concentrations cerebrospinal fluid (CSF) / plasma) and by effectiveness of evacuation system in CNS: P- glycoprotein; breast cancer resistance protein (BCRP) and protein-2 related to multiple drug resistance (MRP-2).

The occurrence of drug neurotoxicity is increased by renal and (or) hepatic insufficiency with disturbance of elimination, medical anamnesis compromised by neuropsychic disorders and background structural anomalies of brain in particular hippocampus sclerosis.

Besides this it's important to assess pharmacokinetic characteristics of drugs like capacity to cross the placental barrier and also to cumulate in breast milk, because the brain influence of drugs could start in antenatal period and continue during breast feeding.

But the principal predisposing factor of drug neurotoxicity in children is the immature but intensively growing brain with the mostly vulnerable period from 6 months of gestation until several initial years of postnatal life [9].

Let's have a closer look at the neurotoxic consequences of use of those drugs that are dedicated firstly to influence CNS - anticonvulsants, antidepressants, antipsychotic drugs and anesthetics.

\section{Anticonvulsants (AC)}

Regardless long-term history of phenobarbital $(>100$ years), phenytoin ( $>75$ years) and inhalation anesthetics (halothane, isofluran) ( $>50$ years) use their impact to the brain development is still poorly investigated. That is why the administration of the mentioned above and other neuroactive drugs during pregnancy and infancy is one of the severe issues in neonatology, neurology and clinical pharmacology [10].

Mentioned above issues are particularly important taking into account the growth of amount of pregnant women suffering from epilepsy and increased quantity of $\mathrm{AC}$ during last 10 years. In USA each year more than 30000 women with epilepsy (from the total number of 1,3 million cases) have got a baby. It's necessary to consider that ACs are used in pregnant women not only for epilepsy but also for other diseases - neuropathic pain, migraine, bipolar psychotic disorders [11].

All ACs are more or less teratogenic. However its teratogenic effect is related mainly to the grand (old) drugs (phenobarbital, phenytoin, carbamazepine and especially valproates) and such effect is decreased or even regressed in new ACs (levetiracetam, lamotrigine, topiramate) $[12,13]$.

In general the incidence of malformations (including CNS) in children born to women who have been taken AC during pregnancy (especially during first trimester of pregnancy) is nearly $4-6 \%$ on the contrary to $2-4 \%$ to women not taken these drugs.

It's necessary to notify that we consider not reasonable (as well as D. Schmidt, 1992) the malformation stratification by syndrome accepted in scientific literature in relation with any anticonvulsant (syndromes related to phenytoin, phenobarbital, carbamazepine) as this categorization is not well specified. In all cases this is a set of the same dysembryogenetic stigmas in various combinations and therefore we would propose a term like "fetal anticonvulsant syndrome" as more appropriate [14].

Apart from the factors described at the beginning of this article the teratogenic risk of ACs (category D by FDA classification) depends in many respects on the drug pharmacokinetic particularities in pregnant woman and fetus. In this relation we would like to distinguish fetal negative and protective factors.

Table 1. Fetal negative and protective factors

\begin{tabular}{|c|c|}
\hline Pregnant woman & Fetus \\
\hline \multicolumn{2}{|l|}{ Fetal negative factors } \\
\hline & Low liver metabolic activity: \\
\hline Polytherapy and high doses of ACs & Diazepam biotransformation descreased at $30-70 \%$ \\
\hline Decreased serum albumin at $20 \% \rightarrow$ increased free fraction of $\mathrm{AC}$ & Decreased pumping ability of endotheliocytes in CNS causing by low \\
\hline $\begin{array}{l}\text { Decrease placenta thickness by the pregnancy termination from } 25 \text { till } 3 \\
\text { micron } \rightarrow \text { facilitation of drug transition to fetus }\end{array}$ & $\begin{array}{l}\text { level of specific drug transporter - P-glycoprotein } \\
\text { Decreased capacity of fetal albumin to bind phenobarbital and diazepam } \\
\text { - increase of its free fractions }\end{array}$ \\
\hline \multicolumn{2}{|l|}{ Fetal protective factors } \\
\hline Pregnant woman & Fetus \\
\hline $\begin{array}{l}\text { Significant increase of hormone activity - induction of liver metabolic activity } \\
\text { following increase } \mathrm{AC} \text { clearance (phenytoin, lamotrigine) }\end{array}$ & $\begin{array}{l}\text { Level of enzymes (CYP 450) per gram of fetal and adult liver is nearly } \\
\text { the same: } 8,6 \pm 1,7 \mathrm{nmol} \text { and } 10 \mathrm{nmol} \text { respectively }\end{array}$ \\
\hline $\begin{array}{l}\text { Increase of renal blood flow with increased elimination of topiramate and } \\
\text { levetiracetam during II and III trimesters of pregnancy }\end{array}$ & Fetal liver consists $4 \%$ of body weight but in adults $-2 \%$ \\
\hline $\begin{array}{l}\text { Phenobarbital and phenytoin are more affinitive to mother's proteins than to } \\
\text { fetal's - decrease entering of these ACs to fetus }\end{array}$ & $\begin{array}{l}\text { CYP } 450 \text { induction in fetal liver in case of phenobarbital intake by } \\
\text { pregnant woman }\end{array}$ \\
\hline
\end{tabular}


Within the conditions of drug intake by pregnant woman placenta is eliminating organ and fetus becomes involuntary recipient, the object of this treatment but its developing brain has low capacity for drugs counteractions.

However pregnant woman and fetus have also counter negative fetal protective mechanisms acting against drugs taken and there are a lot of them.

It is accepted that mentioned fetal protective mechanisms together with skillful doctor's actions are able to prevent or weaken teratogenic action of ACs. It's supposed that there is genotype that is especially sensitive to $\mathrm{AC}$ teratogenic effect. So in accordance with [12] only $6 \%$ of pregnant women with epilepsy who have been treated by several ACs, give birth to children with big malformations (including microcephaly and neural tube defect) and $3.7 \%$ of pregnant women who received monotherapy. Besides teratogenic effect separate cases of neonatal abstinence syndrome were also described for phenobarbital. In addition neonatal abstinence syndrome was notified in neonates whose mothers took oxcarbazepine during third trimester of pregnancy: However not all changes are obvious at birth and within this context it's interesting to perform prospective follow up of children of 3 and 4.5 years old who have been exposed to $\mathrm{AC}$ during antenatal period and did not have big malformations at birth. Concerning this point opinions are divided as it happens.

In accordance with data from Gedzelman E. [14] monotherapy by phenobarbital, phenytoin, carbamazepine, lamotrigine, levetiracetam in the lowest effective dose was not followed by changes of IQ. Lower IQ score (including decrease of working memory) was revealed in valproates and has dose-dependent characteristics. These children need additional education methods.

However in accordance with delayed investigational results cited by Nie Q [10] all ACs (except carbamazepine) have effect at intellectual abilities of children but C. Turski [15] does not exclude also carbamazepine from this group.

It's obvious that cognitive abilities (behavioral teratogenesis) were worse in children after ACs polytherapy in pregnant women especially in case of phenytoin and valproates use, in the contrary the combination of carbamazepine and topiramate or levetiracetam did not lead to the serious complications.

Mechanism: the principal hypothesis explains these disturbances by occurrence of neurons apoptosis and disfucntion of survived neurons caused by AC effect. Confirmation of this hypothesis was obtained at brain MRI ofyoung adults who were exposed to $\mathrm{AC}$ during prenatal period. Minimal morphological changes of grey substance in basal ganglions and hypothalamus were revealed (comparing to the control group of health volunteers of the same age) [14].

\section{Lactation and AC, Psychoactive Drugs (Antidepressants, Antipsychotic)}

\subsection{Anticonvulsants}

Almost all ACs permeate more or less into breast milk if breast feeding woman takes AC regularly. However significant neurotoxicity relates to barbiturates only and manifests by sedative effect: increased somnolence, weakness of suckling, muscular hypotonia, poor body weight gain. Cessation of breast feeding recommended in such case could be followed by child abstinence syndrome: severe irritation, insomnia, tremor, permanent cry. However more sparing recommendations can be used [11]: if ACs are taken in the lowest effective dose it's recommend to skip one breastfeeding act when it corresponds to the maximal drug concentration in blood. And Norse investigators provide at all hopeful results [16]: in women who took ACs (carbamazepine, lamotrigine, phenytoin, valproates) and continued to breast-feed their children up to 6 years old had even higher cognitive abilities and IQ.

\subsection{Antidepressant}

Pregnancy is a serious challenge not only for woman's physical capacities but also for her mental state. It's not coincidence that from $30 \%$ till $50 \%$ of women during pregnancy and during postnatal period suffer from depression of different severity level and therefore antidepressants become one of the most prescribing drugs in pregnant women $[13,17]$. As usual SSRI are considered as drugs of first treatment line, and nearly $6 \%$ of women take these drugs during pregnancy and breast-feeding [18]. In utero fetus is exposed to more severe effect of SSRI than the child during breast-feeding.

\subsubsection{Selective Serotonin Reuptake Inhibitors (SSRI)}

Drug of first choice is paroxetine or sertraline that has low concentration in breast milk and child serum that is associated with minimal adverse effects (AE) - increased irritation, agitation, sleep disturbances, tremor. Even if citalopram seep into maternal milk its concentration in child's blood is very low and clinically not significant. In addition some publications report about somnolence, decreased appetite and low weight in child [19]. It seems that mechanism of these disturbances is in serotonin toxicity serotonergic hyperstimulation syndrome. In spite of possible adverse reactions related to SSRI refusal from treatment of depression will result in worst consequences and breastfeeding stop will aggravate them [18].

Fluoxetine is not a drug of choice during lactation period due to long-term half-life period (especially its active metabolite) and due to ability to cumulate in newborn's blood that is threatening for severe neurological complications (coma in child whose mother took this drug during pregnancy and lactation period) [19].

\subsubsection{Tricyclic Antidepressants (TCA)}

In TCA group nortriptyline and imipramine are the most investigated and safe drugs that have next to none concentrations in baby's blood. It is not recommended to take doxepine for breastfeeding woman as it has metabolite with long-term effect that could cause significant sedative effect in 
child and breath depression.

\subsection{Antipsychotic Drugs}

Drugs of first (I) generation - typical antipsychotics (e.g. chlorpromazine and fluphenzine) could cumulate causing sedative effect in child on breast feeding with restricted data about long-term complications. Haloperidol is considered as quite safe drug however it's notified retardation of psychomotor development in some cases.

Atypical antipsychotic drugs - II generation. Clozapine (due to its ability to cumulate in breast milk in case of longterm intake and to cause increased somnolence, speech development delay and even seizures) and risperidone (due to high risk of extrapyramidal disturbances) are not recommended for use by breastfeeding women [13]. In addition to that in some publications it reports about use of risperidone by breastfeeding women without any events [20].

Data concerning olanzapine are ambiguous but it's considered that its administration risk could exceed the benefit during lactation period.

Quetiapine (in monotherapy) is considered quite safe as it has low concentration in breast milk and therefore in child's blood that is explained its good tolerance [19].

\section{Anesthetic Drugs of Local and General Application}

In case of long general anesthesia of parturient woman having pathological childbirth process newborn could develop CNS depression syndrome and disadaptation during first 24 hours ("anesthetic child"). When using intravenous injection of anesthetic drugs (propofol, ketamine) its elimination depends on liver biotransformation and renal excretion. Because of both functions are not completely developed in neonates (especially in premature newborns) so time required for elimination of these drugs (and therefore for withdrawal from anesthesia) could be longer than in case of inhalation drugs use - nitrous oxide, sevoflurane, halothane [14].

At the same time single and short-duration (less than one hour) administration (e.g. during Caesarian operation) of intravenous or inhalation anesthetics could not more likely cause severe neurotoxic effects [19].

However FDA warns [21] that sedation or general anesthesia during more than 3 hours or repeated for several times during critical period of brain development (last trimester of pregnancy and first three years of postnatal life) are associated with disturbances of cognitive functions (longterm memory, educability) in child that is caused by partial apoptosis of neurons, disturbances of synaptogenesis and possible by myelinogenesis [22].

The concept of local anesthetics (lidocaine, ropivacaine) that they have only local action has to be abolished due to cumulated data about its ability for systemic effect including neurotoxic action mainly as seizures. It's related to its significant absorption from the site of injection in case of overdose and especially in case of penetration into vessel or during neck and head surgery [5, 23, 24].

The group of increased risk for development of neurotoxicity includes pregnant women and children of first year of life mainly newborns who have slowed metabolism (decrease hydroxylation, for example, of lidocaine) and elimination of anesthetics. In addition they demonstrate decreased plasma concentration of alpha-1-acid glycoprotein and its insufficient ability to bind anesthetics following by increase of its free fraction [24].

As long as neurogenesis in some parts of the brain continues throughout life the negative effect of drugs does not stop with the end of critical period of brain development. So in accordance with $[25 ; 26]$ the clinical manifestation of psychoactive drug abuse in children of school age in $76.2 \%$ is characterized by decreased intellectual-mnestic ability. Anticonvulsants have more different manifestations of neuropsychic disturbances with phenobarbital as a leader of the group but also other "old" well known drugs phenytoin, valproic acid, carbamazepine. It's considered that modern drugs - lamotrigine, vigabatrin, gabapentin, topiramate - have less neurotoxic potential. In addition neuropsychotic disturbances often have transitory characteristics occurring during titration period. Suppressing epileptiform activity ACs also act at those parts of the brain which are involved in memory process, education and regulation of emotional reactions through gamma-aminobutyric acid (GABA), serotonin and antiglutamatergic mechanisms [11].

Unfortunately neurotoxicity does not limit by intake of only neuroactive drugs. There is quite long list of drugs that have side effects (of different degree and type) like disturbances of central or peripheral nervous system. Let's pay more attention to some drug groups from this list.

In accordance with Italian authors [27] the majority of adverse drug reactions (including neurotoxicity) develop in case of antibiotics (AB) use. Possibly it's not occasional because many of them (e.g. $\beta$-lactams and fluoroquinolones) are used for meningitis treatment and therefore have to well pass blood-brain barrier (BBB) and cumulate in CSF and brain [28].

\section{6. $\beta$-lactams}

There are various manifestations of neurotoxicity in this group of antibiotics: enchephalopathy, disturbances of mental status, myoclonus, seizures, flapping tremor, non-convulsive epileptic status.

Mechanism:

a) Concentration-dependent concurrent antagonism of brain $\gamma$-aminobutyric acid (GABA) - main inhibitor of neurotransmitter

b) Induction of cytokines release including TNF- $\alpha$ that could have direct cerebral toxicity

c) Increase glutamatergic activity

Predisposing factors:

a) High dosage

b) Renal insufficiency with increased CSF / blood 
concentration ratio up to $34-45 \%$ comparing to normal $10 \%$

c) Background CNS anomalies [29, 30].

\section{Fluoroquinolones}

Variety of neuropsychic changes in case of use of this $\mathrm{AB}$ group is also rather wide: transitory psychosis, insomnia, anxiety, depression, headache, very rare $(0.9-2 \%)$ convulsive reactions predominantly in patients with convulsive readiness or as consequences of interaction with other drugs (euphyllin, imipenem, metronidazole).

It's proposed that the mechanism of such complications develops through counteraction of fluoroquinolones to binding of $\gamma$-aminobutyric acid with its receptor.

It is noticed that fluroquinolones ability to enter into CNS does not always correlate with potential epileptogenicity. For instance, ofloxacin with SCF concentration equal to $50 \%$ from its serum concentration is less neurotoxic that ciprofloxacin that has the same parameter less by half [31].

\section{Drugs from Other Therapeutic Groups}

It was mentioned above about the role of age-dependent, genetic factors and drug dose in the development of neurotoxicity. Here are several examples (like reminder) of drugs from other therapeutic groups.

Naphazoline (naphthyzin) - drug for rhinitis treatment - is prohibited for children under 1 year old in concentration $0.05 \%$ and under 18 years old in concentration $0.1 \%$. Because of resorption from nasal cavity (due to mistaken use in children under one year old) naphazoline has significant neurotoxic effect - severe CNS depression (own nonpublished data - children of 5 and 10 months).

Propofol is narcotic non-inhalation drug for anesthesia support and sedation for patients at artificial lung ventilation (ALV). It's considered as ideal drug for anesthesia due to fast effect (in 30-60 seconds) and support of sedation during all infusion period. However using of dose above $4 \mathrm{mg} / \mathrm{kg} /$ hour and duration of infusion more than 48 hours are followed by rhabdomyolysis, renal insufficiency and consciousness loss until deep coma [32].

Vincristine is anti-tumor drug of herbal origin. It has specific pharmacokinetic characteristics like active liver biotransformation with enzymes of cytochrome P450 system - CYP3A4 and CYP3A5. In people from Caucasia in Russia there is high frequency $(80 \%)$ of polymorphism of CYP3A5 with limited CYP3A4 activity in children of tender age with abrupt decline of vincristine metabolism in case of its use following by visceral neuropathy - paralytic ileus [33, 34].

In all cases mentioned above neurotoxicity was caused by mistaken non-intentional actions of parents (naphazoline) or doctors (propofol, vincristine) as well as due to lack of knowledge.

However it is known cases of compulsive well-considered use by mother of the drugs for child, for instance amitriptyline [35] or carbamazepine (own non-published data) with severe neurotoxic consequences (seizures, consciousness loss) for creation of impression of child's illness - syndrome Munchausen by proxy.

\section{Conclusion}

Thus neurotoxicity is not rare case while using drugs of different therapeutic classes and it varies from visceral neuropathy and neuromuscular blockage up to consciousness loss, seizures, non-specific encephalopathy with decrease of memory, educability and IQ.

Population risk of neurotoxicity associated with different classes of drug includes certain age groups (fetus, newborns, children of first years of life), severity of disease, ethnic and genetic peculiarities, liver or renal insufficiency, background neuropsychic disorders.

Awareness of this information helps to avoid or facilitate in some cases occurring of such severe drug side effect as neurotoxicity.

\section{Conflict of Interest}

All the authors do not have any possible conflicts of interest.

\section{References}

[1] Mira Harrison-Woolrich, Juan Garcia-Quiroga, Jahella Ashton and Peter Herbison "Safety and Usage of Atypical Antipsychotic Medicines in Children” Drug Safety, 2007; 30 (7): $569-79$

[2] Stephen Toovey, Craig Rayner, Eric Prinssen et al "Assessment of Neuropsychiatric Adverse Events in Influenza Patients Treated with Oseltamivir: A Comprehensive Review”. Drug Safety, 2008; 31 (12): 1097-1114.

[3] Stephanie Cham, Hayley J. Koslik, Beatrice A. Golomb "Mood, Personality, and Behavior Changes During Treatment with Statins: A Case Series". Drug Safety - Case Reports. December 2016, 3: 1-13.

[4] Kwan P., Brodie MJ «Neuropsychological effects of epilepsy and antiepileptic drugs» The Lancet v. 357, № 9251. January 2001.

[5] Rezvani M, Finkelstein Y, Verjee Z, Railton C, Koren G "Generalized seizures following topical lidocaine administration during circumcision: establishing causation". Paediatric drugs, 2007; 9 (2): 125-127.

[6] Marco Mula, Josemir W. Sander "Negative Effects of Antiepileptic Drugs on Mood in Patients with Epilepsy". Drug Safety, July 2007, Volume 30, Issue 7, pp 555-567.

[7] James E Tisdale, Douglas A. Miller "Drug induced diseases". American Society of Health-system Pharmacists. Bethesda, 2010, p 179-316.

[8] Bleggi-Torres LF, de Medeiros BC, Werner B. "Neuropathological findings after bone marrow transplantation: an autopsy study of 180 cases". Bone Marrow Transplant. 2000; 25 (3): 301. 
[9] Patrick A. Forcelli. "Short- and Long-Term Neurological and Psychiatric Sequelae of Developmental Exposure to Antiepileptic and Anesthetic Drugs". Front Neurol. 6: 41, 2015.

[10] Nie Q, Su B, Wei J et al. "Neurological teratogenic effects of antiepileptic drugs during pregnancy". Exp Ther Med. 2016 Oct; 12 (4): 2400-2404. Epub 2016; Aug 29.

[11] O. A. Pylaeva, K. Y. Mukhin, A. S. Petrukhin « Side effects of antiepileptic therapy ». Publishing house "Garant", 2016.

[12] L. V. Ushkalova, E. A. Ushkalova «Safety of psychotic drugs during lactation ». Pharmateka 2013, N1, pp 55-63.

[13] Payne JL. Psychopharmacology in Pregnancy and Breastfeeding. Med Clin North Am. 2019 Jul; 103 (4): 629650 .

[14] Gedzelman E, Meador K. "Neurological and psychiatric sequelae of developmental exposure to antiepileptic drugs". Front Neurol (2012) 3: 182. doi: 10.3389/fneur.2012.00182.

[15] Turski CA, Ikonomidou C. "Neuropathological sequelae of developmental exposure to antiepileptic and anesthetic drugs". Front Neurol (2012) 3: 120. doi: 10.3389/fneur.2012.00120.

[16] Johnson EL, Burke AE, Wang A, Pennell PB. Unintended pregnancy, prenatal care, newborn outcomes, and breastfeeding in women with epilepsy. Neurology. 2018 Sep 11; 91 (11): e1031-e1039.

[17] Korotaeva N. V., Ippolitova L. I., Nastausheva T. L., Ivanova O. A., Kogutnitskaya M. I., Pershina E. S. Psychological features of mothers who have given birth to premature infants. Rossiyskiy Vestnik Perinatologii i Pediatrii (Russian Bulletin of Perinatology and Pediatrics). 2019; 64 (4): 38-44. (In Russ.)

[18] Weisskopf E, Fischer CJ, Bickle Graz M, Morisod Harari M, Tolsa JF, Claris O, Vial Y, Eap CB, Csajka C, Panchaud A. Risk-benefit balance assessment of SSRI antidepressant use during pregnancy and lactation based on best available evidence. Expert Opin Drug Saf. 2015 Mar; 14 (3): 413-27.

[19] Wainer C. P., Mason C. Drugs for pregnant and lactating women. Third edition, 2019. Elsvier.

[20] Anderson KN, Ailes EC, Lind JN, Broussard CS, Bitsko RH, Friedman JM, Bobo WV, Reefhuis J, Tinker SC; National Birth Defects Prevention Study. Atypical antipsychotic use during pregnancy and birth defect risk: National Birth Defects Prevention Study, 1997-2011. Schizophr Res. 2019 Nov 21

[21] Brown R. E., Agarwal R. AAP responds to FDA warning jn anesthesia use in children. AAP News, 2017.

[22] Creeley, C. E. From Drug-Induced Developmental Neuroapoptosis to Pediatric Anesthetic Neurotoxicity-Where Are We Now? Brain Sci. 2016, 6, 32.

[23] Postnikov S. S., Kostyleva M. N., Linkova T. V., Lopatin A. $\mathrm{V}$. "Usual and unusual side effects of ropivacaine (naropine) in child of 6 months old". Children Hospital 2009; 3 (37), pp. 19-21.

[24] El-Boghdadly K, Pawa A, Chin KJ. Local anesthetic systemic toxicity: current perspectives. Local Reg Anesth. 2018 Aug 8; 11: 35-44.

[25] Zeljko J Bosnjak, Sarah Logan, Yanan Liu "Recent Insights Into Molecular Mechanisms of Propofol-Induced Developmental Neurotoxicity: Implications for the Protective Strategies" Anesthesia and analgesia August 2016; 123 (5): 1286-1296.

[26] Kashirskaya E. I. «Clinical biochemical assessment and forecast for health of children who develop under psychoactive drugs influence». Summary of doctoral dissertation. Astrakhan, 2010.

[27] Ferrajolo C, Capuano A, Trifiro G. "Pediatric drug safety surveillance in Italian pharmacovigilance network: an overview of adverse drug reactions in the years 2001-2012". Expert opinion on drug safety. 2014; 13 Suppl 1: S9-20. Epub 2014/08/30. doi: 10.1517/14740338. 2014.939581PMID: 25171155 .

[28] Rama K Maganti. "Neurotoxic effects associated with antibiotic use: Management considerations". British Journal of Clinical Pharmacology 72 (3): 381-93 April 2011.

[29] Charlotte Durand-Maugard, Anne-Sophie Lemaire-Hurtel, Valérie Gras-Champel "Blood and CSF monitoring of cefepime-induced neurotoxicity: Nine case reports". Feb 2012. Journal of Antimicrobial Chemotherapy, 67 (5): 1297-9.

[30] Ki Bae Kim, Sun Moon Kim, Woori Park et al. "CeftiaxoneInduced Neurotoxicity: Case Report, Pharmacokinetic Considerations, and Literature Review". Journal of Korean medical science 27 (9): 1120-3 · September 2012.

[31] E. N. Padeyskaya "New fluoroquenolones: successes and failures". In the book "Gold pages". Moscow, 2016, pp. 23543.

[32] S. S. Postnikov, M. N. Kostyleva, G. P. Brusov. "Case of infusion syndrome in child of 10 years old". Safety and risk in pharmacotherapy. - 2016. - N4, pp. 5-10.

[33] Renbarger JL, McCammack KC, Rouse CE, Hall SD. "Effect of race on vincristine associated neurotoxicity in pediatric acute lymphoblastic leukemia patients" Pediatric Blood and Cancer 2008 April; 50 (4): 769 - 71. PMID: 18085684.

[34] Postnikov S. S., Strykov V. A., Kostyleva M. N., Ilina E. S., Gracianskaya A. N., Strock A. B. The case of dynamic intestinal obstruction, caused by an individual intolerance to vincristine in a child of 5 years. Safety and Risk of

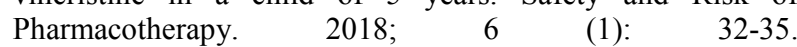
https://doi.org/10.30895/2312-7821-2018-6-1-32-35.

[35] Manikoth P., Subramanyan R., Menon S., Al Khusaiby S. M. «A child with cardiac arrhythmia and convulsions» The Lancet, v354, December 11, 1999, p 2046. 